\title{
Thin film magnetoelectric composites near spin reorientation transition
}

\author{
N. Tiercelin ${ }^{\mathrm{a}, *}$, V. Preobrazhensky ${ }^{\mathrm{a}, \mathrm{b}}$, V. Mortet $^{\mathrm{c}}$, A. Talbi $^{\mathrm{a}}$, A. Soltani ${ }^{\mathrm{d}}$, K. Haenen ${ }^{\mathrm{c}}$, P. Pernod ${ }^{\mathrm{a}}$ \\ a Joint European Laboratory LEMAC: IEMN CNRS 8520-Ecole Centrale de Lille, Villeneuve d'Ascq 59652, France \\ b Joint European Laboratory LEMAC: WRC-GPI-RAS 117454, Vernadsky prosp. 78, Moscow, Russia \\ c Institute for Materials Research (IMO), Hasselt University, IMEC vzw, Division IMOMEC, B-3590 Diepenbeek, Belgium \\ d IEMN CNRS 8520, Bd Poincaré, Villeneuve d'Ascq 59651, France
}

\section{A R T I C L E I N F O}

Available online 14 February 2009

Keywords:

Stress mediated magnetoelectric effect

Thin film

Nanostructured magnetostrictive

multilayer

Spin reorientation transition

\begin{abstract}
A B S T R A C T
We report the use of a magnetic instability of the spin reorientation transition type to enhance the magnetoelectric sensitivity in magnetostrictive-piezoelectric structures. We present the theoretical study of a clamped beam resonant actuator composed of a piezoelectric element on a passive substrate actuated by a magnetostrictive nanostructured layer. The experiments were made on a polished $150 \mu \mathrm{m}$ thick $18 \times 3 \mathrm{~mm}^{2}$ lead zirconate titanate (PZT) plate glued to a $50 \mu \mathrm{m}$ thick silicon plate and coated with a giant magnetostrictive nanostructured $\mathrm{Nx}\left(\mathrm{TbCO}_{2} \mathrm{~nm} / \mathrm{FeCO}_{5 \mathrm{~nm}}\right)$ layer. A second set of experiments was done with magnetostrictive layer deposited on PZT plate. Finally, a film/film structure using magnetostrictive and aluminium nitride films on silicon substrate was realized, and showed ME amplitudes reaching $30 \mathrm{~V} \mathrm{Oe}^{-1} \mathrm{~cm}^{-1}$. Results agree with analytical theory.
\end{abstract}

(c) 2009 Elsevier B.V. All rights reserved.

\section{Introduction}

The magnetoelectric (ME) effect, predicted by Curie in 1894 is the change of electric polarization as a response to an applied magnetic field $[1,2]$. With the development of new techniques, the interest for ME effect grew again (see Fiebig's reviews $[3,4]$ ): new "stress mediated" composite materials mainly associating magnetostrictive and piezoelectric or electrostrictive based materials were created and displayed ME effects several orders of magnitude higher than in natural ME materials. ME coefficient values as high as $10 \mathrm{VOe}^{-1} \mathrm{~cm}^{-1}$ could be obtained in TerfenolD/PMN-PT laminate composites. Higher values can be found in the case of resonant devices [5-8]. Most of these composites are still bulk materials, and therefore, hard to integrate in micro-electromechanical-systems (MEMS) or microelectronic devices. Recent works on ME epitaxial nanostructures show very promising features [9]. In the present work, we investigated RF-sputtered thin film based solutions combined with the use of a magnetic instability which highly increases the sensitivity of the magnetomechanical interaction [10] and as a result enhancement of ME effect.

\section{Spin reorientation transition in giant magnetostrictive nanostructured multilayers}

The magnetic multilayer technology enables the preparation of nanostructures with tailored magneto-elastic properties. In

\footnotetext{
* Corresponding author. Tel.: +33320197946; fax: +333201978 78

E-mail address: Nicolas.Tiercelin@iemn.univ-lille1.fr (N. Tiercelin).
}

particular, the $\left(\mathrm{TbFe}_{2} / \mathrm{Fe}\right)$ or $\left(\mathrm{TbCO}_{2} / \mathrm{FeCo}\right)$ nanostructured multilayers with giant magnetostriction and almost perfect uni-axial magnetic anisotropy were elaborated $[11,12]$. These structures are of interest because of brightly expressed spin reorientation transition (SRT) in a transversal bias magnetic field. In the vicinity of the SRT the magnetic subsystem becomes strongly nonlinear and anomalously sensitive to the external driving field [13-17]. Integration of these materials into MEMS has been shown $[18,19]$. For a given uni-axial magnetostrictive layer with a magnetic configuration such as the one shown in Fig. 1, the magnetic free energy of the system can simply be written as the sum of the Zeeman energy and the anisotropy energy restricted at its first term:

$F=-M\left(H_{S} \cos (\varphi)+h \sin (\varphi)+\frac{1}{2} H_{A} \sin ^{2}(\varphi)\right)$

where $H_{A}$ is the in plane anisotropy field. This can then be used to understand the behaviour of the magnetization angle $\varphi$.

When the bias field $H_{S}$ is perpendicular to the easy axis (EA) and strong enough (i.e. $H_{S}>H_{A}$ ), magnetization is parallel to $H_{S}$ and this orientation is stable. Application of an alternating field with an amplitude of a few Oersteds perpendicularly to $H_{S}$ leads to a linear oscillation of the magnetization with a small angular amplitude. The magneto-elastic vibrations are linearly excited in such conditions. In the point of SRT $\left(H=H_{A}\right)$, the magnetic subsystem becomes unstable: thanks to the flat shape of the free energy, the amplitude of magnetic oscillations increases (Fig. 2) and the response to $h$ is strongly nonlinear. When $H_{S}<H_{A}$, the equilibrium angle of the magnetization is rotated towards the anisotropy axis (angular phase) and linear oscillation around this position takes place when for low amplitudes of $h$. As expected for 


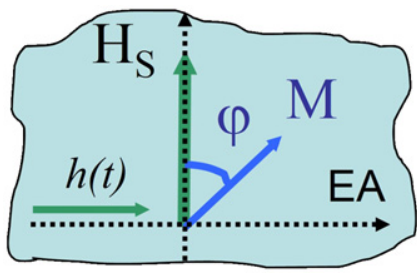

Fig. 1. Magnetic configuration of the uni-axial multilayer. Orientations of the bias $H_{S}$ and alternating $h$ magnetic fields and magnetization $M$ relatively to the easy axis (EA) are shown.

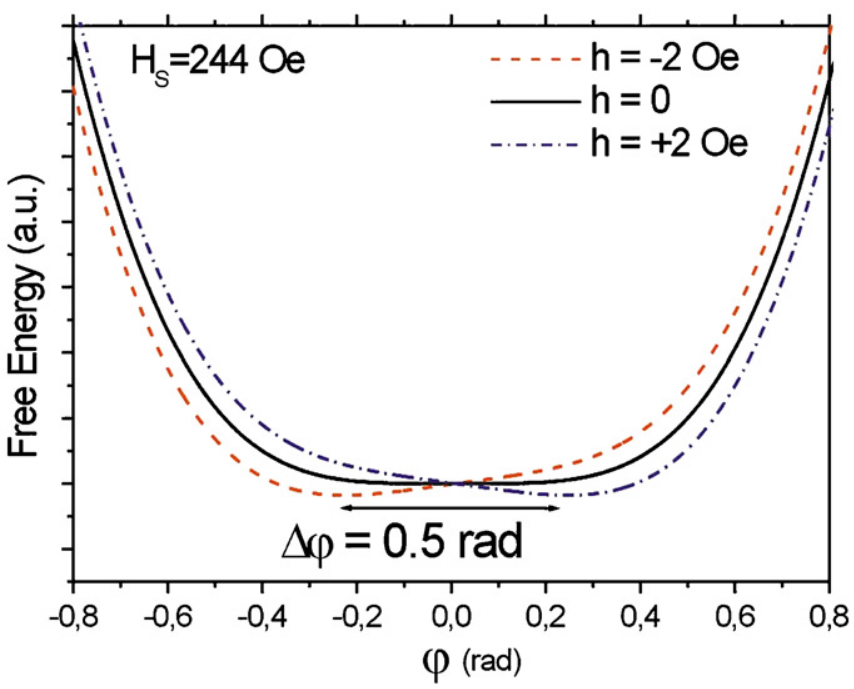

Fig. 2. Free energy of the system as a function of the magnetization angle $\varphi$, calculated at the SRT point. Calculations were made here with a value of $H_{A}=2440$ e and an alternative excitation amplitude of 20 . The resulting magnetization angle amplitude is about 0.5 rad.

the SRT, this kind of behaviour is consistent with the Landau theory of second order phase transitions [20].

In the case of giant magnetostrictive films, the maximum deformation of the material is obtained when the magnetization rotates homogeneously with a $90^{\circ}$ angle in the plane of the layer. Thus, the extreme sensitivity of the magnetic system at the SRT point is reflected on the magneto-elastic system and can be, in turn, exploited in stress mediated hybrid magnetostrictive/piezoelectric ME materials.

\section{Theory of ME effect for vibrating substrate/film structures}

With the configuration given in Fig. 3, we already showed [21] that for a given resonance mode number $n$, the amplitude of ME voltage generated by an excitation field $h$ at a frequency $\omega$ could be written as

$$
\begin{aligned}
V= & \frac{2 B\left(e_{31}-e_{33} C_{12} / C_{11}\right)}{\varepsilon \rho\left(\Omega_{n}^{2}-\omega^{2}+2 i \delta_{n} \omega\right)} \frac{d_{m} d_{p}}{4 d}\left(2 d_{1}-d_{m}\right)\left(2 d_{1}-2 d_{m}-d_{p}\right) \\
& \times \frac{f_{n} \chi h}{S^{2}}
\end{aligned}
$$

with the dimensionless function $f_{n}=S \mu_{n} \gamma_{n} / \int U_{n}^{2} d S$.

$B$ is the magnetomechanical coupling constant, $h$ is the magnetic excitation field, $\chi=\partial \varphi / \partial h$ is the sensitivity of the magnetization angle $\varphi$ with respect to $h, A_{n}$ is the amplitude of vibration of the considered mode.

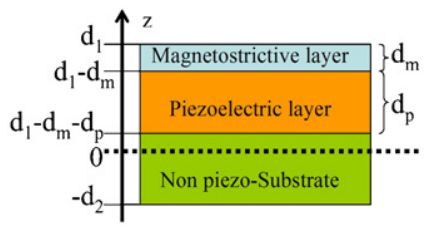

Fig. 3. Side schematic view of the considered magnetostrictive actuator with integrated piezoelectric material stacked on a non-piezoelectric (passive) substrate.

$\mu_{n}$ and $\gamma_{n}$, respectively, are the magnetostrictive and piezoelectric form factors that can be expressed as

$\mu_{n}=\iint\left(\frac{\partial^{2} U_{n}}{\partial x^{2}}-\frac{\partial^{2} U_{n}}{\partial y^{2}}\right) d S$

for bending vibration modes,

$\mu_{n}=\iint\left(2 \frac{\partial^{2} U_{n}}{\partial x \partial y}\right) d S$

for torsion vibration modes,

$\gamma_{n}=\iint\left(\frac{\partial^{2} U_{n}}{\partial x^{2}}+\frac{\partial^{2} U_{n}}{\partial y^{2}}\right) d S$

where $U_{n}(x, y)$ represents the envelope of the elastic displacement along $z$ axis for the considered vibration mode. The $e_{i j}$ are the piezoelectric constants, $C_{i j}$ are the elastic moduli, $\delta_{n}$ is the damping coefficient, $\Omega_{n}$ is the renormalized resonance frequency, $\rho$ the substrate density and $\varepsilon$ is the dielectric coefficient of the piezoelectric layer.

From this, it can be calculated that for the film/film ME layer on a thicker substrate, bending vibration modes can result in ME voltage whereas torsion vibration will result in no effect. It can be also deduced that if there is a thin magnetic film on a thick piezoelectric layer and no substrate, neither bending non torsion vibration will lead to generation of ME voltage.

In the case of 'in plane' modes, such as longitudinal vibration modes, similar calculations can be done leading to

$V=\frac{d_{m} d_{p}}{d} \frac{B\left(e_{31}-e_{33} C_{12} / C_{11}\right)^{2}}{\varepsilon \rho} \frac{f_{n}}{\left(\Omega_{n}^{2}-\omega^{2}+2 i \delta_{n} \omega\right)} \chi h$

with

$f_{n}=\mu_{n} \gamma_{n} / S \cdot \int\left(U_{x}^{n 2}+U_{y}^{n 2}\right) d S$

Magnetostrictive and piezoelectric form factors $\mu_{n}$ and $\gamma_{n}$, respectively, are, for a longitudinal vibration, mode

$\mu_{n}=\int d S\left(\frac{\partial U_{x}^{n}}{\partial x}-\frac{\partial U_{y}^{n}}{\partial y}\right)$ and $\gamma_{n}=\int d S\left(\frac{\partial U_{x}^{n}}{\partial x}+\frac{\partial U_{y}^{n}}{\partial y}\right)$

This time $U_{x}^{n}$ and $U_{y}^{n}$ are the envelopes of elastic displacement along the $x$ and $y$ axes of the vibration mode $n$. Other parameters are the same as previously defined.

The expression of $V$ in this case shows that for a cantilever type device (beam clamped at one end), the first longitudinal vibration mode will lead to generation of ME voltage.

\section{Experiments and discussion}

Three configurations have been realized and characterized. 
4.1. Magnetostrictive nanostructure with SRT on thick PZT layer and passive substrate

In our experiments, the considered actuator is a polished $150 \mu \mathrm{m}$ thick $18 \times 3 \mathrm{~mm}^{2}$ commercial lead zirconate titanate (PZT) plate with an out of plane polarization, coated with a nanostructured giant magnetostriction film composed of 50 bilayers of $\mathrm{TbCO}_{2}(5 \mathrm{~nm}) / \mathrm{FeCo}(5 \mathrm{~nm})$, deposited by RF-sputtering. The magneto-elastic constant was measured using the cantilever method [22] and was found to be $b^{\gamma, 2} \approx-7 \mathrm{MPa}$. A magnetic EA is obtained in the magnetic layer by applying a magnetic field during deposition. This layer also serves as the top electrode, whereas the original bottom electrode of the PZT beam was kept. This part is then glued with diluted silver epoxy glue to a $50 \mu \mathrm{m}$ silicon plate previously coated with a platinum layer to ensure the electrical connection of the bottom electrode. The glue thickness is negligible compared to the PZT and silicon, and is considered as a part of the passive substrate. Fig. 4 shows the considered device along with the magnetic field configuration used in the experiments.

For this device, the EA was oriented at $45^{\circ}$ with respect to the length of the beam. In this configuration, magneto-elastic bending vibrations are favoured [23].

For the characterizations, an electromagnet is used to apply the SRT polarizing field $H_{S}$, and the alternative driving field $h(t)$ is generated by a 200 windings coil. The ME voltage picked between bottom and top electrodes of the PZT is fed to a digital oscilloscope through a high impedance charge amplifier (voltage amplification equal to one). The bending and torsion vibration amplitudes of the cantilever are recorded using a similar method as in Ref. [22]. A two-dimensional position sensitive diode (PSD_Hamamatsu Photonics) measures the deflection of a laser beam reflected on the free tip of the cantilever. Fig. 3 shows the ME voltage amplitude measured as a function of the magnetic polarizing field $H_{S}$, when exciting the actuator at $177 \mathrm{~Hz}$ with a $h_{0}=30$ Oe $(3 \mathrm{mT})$ magnetic field amplitude. Using the optical detection part of the experimental setup, this mode has been identified as the first flexural mode of the actuator. For this mode and this geometry, the magnetostrictive and piezoelectric form factors $\mu_{1}$ and $\gamma_{1}$ calculated with the analytical expression of the vibration deformation define $f_{1}$ as approximately equal to 0.21 . It means that this vibration mode is compatible with the ME effect. As a comparison, for the first torsion mode, in this geometry, $\gamma$ is equal to zero, leading to no ME signal.

The effect of the SRT can be clearly seen in Fig. 5: When $H_{S}$ reaches the anisotropy field value of the magnetic layer, the SRT takes place, magneto-elastic efficiency is enhanced, and so is the $\mathrm{ME}$ coefficient. Using this effect, a $580 \mathrm{mV} \mathrm{Oe}^{-1} \mathrm{~cm}^{-1}$ value could

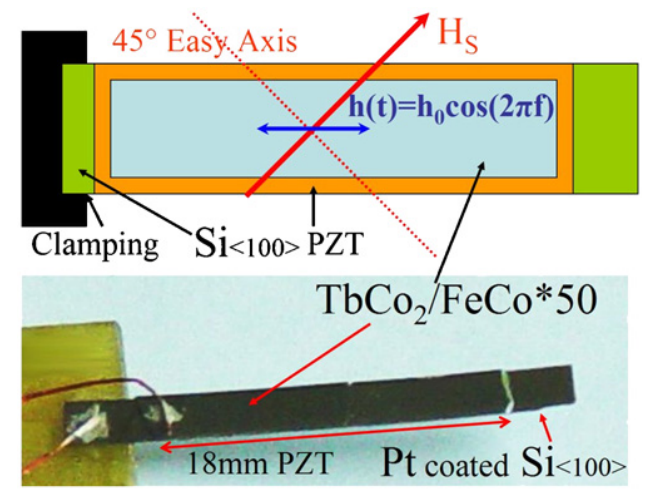

Fig. 4. Top: magnetic field configuration for the clamped PZT beam coated with an active magnetic film and placed on a silicon substrate. $H_{s}$ is the polarizing field. $h(t)$ is the excitation field. Bottom: actual device.

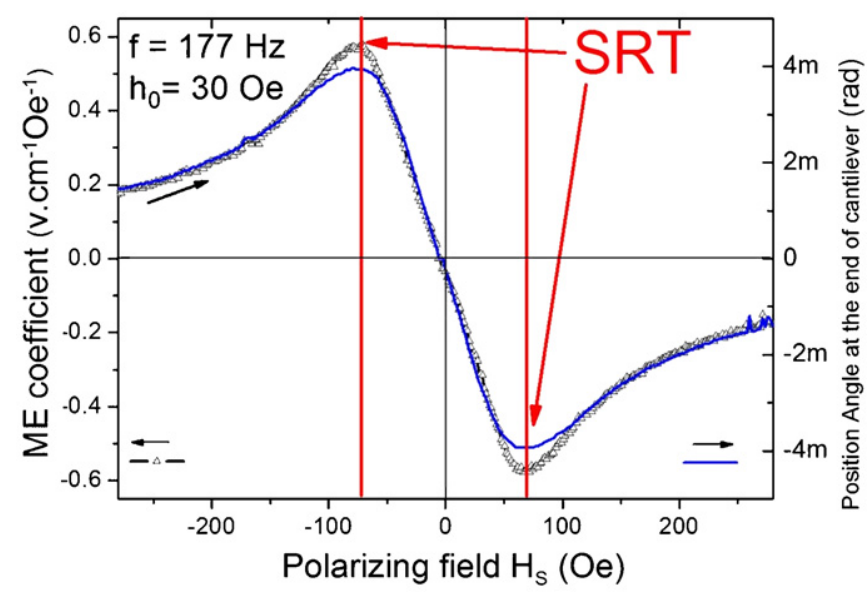

Fig. 5. Measured ME coefficient (triangles) and bending angular position (solid line) for the first resonance mode of a PZT on substrate clamped beam. The phase of the signals is taken into account. Thickness of the PZT was $150 \mu \mathrm{m}$ on a $50 \mu \mathrm{m}$ silicon substrate.

be achieved in resonant mode. This value is obtained for a $500 \mathrm{~nm}$ active magnetic layer being 300 times thinner than the PZT substrate. The angle amplitude at the end of the actuator measured by the optical setup shows a fairly good agreement with the magnetic field dependence of the ME voltage. The phases of the signals are also synchronized. Thus, the ME voltage can be used as an indicator of the actuator position. One can also note that for $H_{S}$ equal zero, both ME signal and vibration amplitude are not equal to zero: this is due to the hysteresis of the magnetic layer.

\subsection{Magnetostrictive nanostructure with SRT on piezoelectric substrate}

We investigated the results given by a simple PZT beam, without the passive substrate underneath. The considered actuator is a polished $480 \mu \mathrm{m}$ thick $30 \times 3 \mathrm{~mm}^{2}$ commercial PZT plate with an out of plane polarization coated with a giant nanostructured magnetostrictive film composed of 50 bilayers of $\mathrm{TbCo}_{2}(5 \mathrm{~nm}) / \mathrm{FeCo}(5 \mathrm{~nm})$, as in the first device. From theory, it can be seen that if there is no substrate under the piezoelectric layer (i.e. $-d_{2}=d_{1}-d_{m}-d_{p}$ ) and the magnetostrictive layer is thin, then, the term $2 d_{1}-2 d_{m}-d_{p}$ is equal to zero. In this case, for the bending resonance mode, the neutral fibre is at half the height of the actuator, and stresses in the upper part cancel those in the lower part resulting in no piezoelectric voltage, contrary to the quasistatic case considered in Ref. [24].

Fig. 6 shows the magnetoelectric voltage amplitude measured as a function of the magnetic polarizing field $H_{S}$, when exciting the actuator at $17.93 \mathrm{KHz}$ with a $3 \mathrm{mT}$ magnetic field amplitude. This mode has been identified as the first longitudinal mode of the actuator: no bending or torsion angle could be measured optically. For this mode, contrary to bending and torsion, stresses inside PZT do not cancel each other out. As predicted by the theoretical model for longitudinal mode, ME voltage is generated. It appears that despite much smaller absolute displacements of the beam, the measured voltage amplitude is of the same order of magnitude as in the case of flexural vibration modes. The effect of the SRT can also be clearly seen here. In this case, there is a slight hysteresis caused by the meta-stability of the domain structure in the film changing the apparent position of the critical field for SRT when $H_{S}$ goes up or down. In resonant mode $100 \mathrm{mV} \mathrm{Oe}^{-1} \mathrm{~cm}^{-1}$ could be achieved. In this configuration, the 


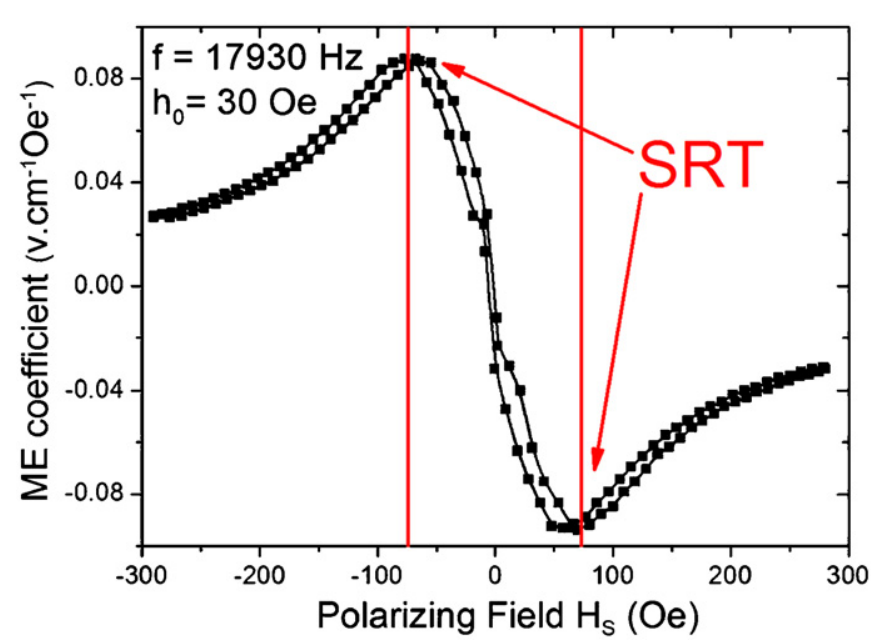

Fig. 6. ME voltage for the first longitudinal mode as a function of the polarizing field $H_{S}$. The phase of the signal is taken into account.

$500 \mathrm{~nm}$ active magnetic layer is 1000 times thinner than the PZT substrate.

\subsection{Magnetostrictive nanostructure with SRT on aluminium nitride (AlN) thin film on silicon substrate}

The considered device is a polished $50 \mu \mathrm{m}$ thick $18 \times 3 \mathrm{~mm}^{2}$ silicon $\langle 100\rangle$ substrate, on which a platinum electrode is deposited, and subsequently coated with AlN followed by the giant magnetostrictive nanostructured layer, both deposited by radio frequency (RF) sputtering technique.

Piezoelectric AlN films were deposited by reactive DC-pulsed magnetron sputtering under optimized deposition conditions. The study of the deposition conditions were described elsewhere [25]. An aluminium target ( 4 in in diameter, $99.99 \%$ purity), which is held on the water-cooled magnetron cathode, is sputtered in a mixture of $50 \%$ argon and $50 \%$ nitrogen. The distance between the target and the copper substrate holder is adjusted at $5 \mathrm{~cm}$. The substrates are not heated and their temperature is only dependent on the plasma heating. Before deposition, the sputtering chamber was evacuated to a pressure below $2 \times 10^{-4} \mathrm{~Pa}$. The target power supply is driven in constant-power mode at $600 \mathrm{~W}$ with a $250 \mathrm{kHz}$ pulse frequency and $1600 \mathrm{~ns}$ pulse width. Before deposition, the target is cleaned in argon plasma for $10 \mathrm{~min}$ and it is pre-sputtered for an additional $10 \mathrm{~min}$. During these steps, the substrates are shielded from deposition by a shutter. Under optimized deposition conditions, smooth, polycrystalline and low stress AlN films with (002) texture and a columnar structure are obtained. In order to avoid short circuits, some photoresist (shipley S1818) was manually laid along the edges of the substrate. The giant nanostructured magnetostrictive film composed of 50 bilayers of $\mathrm{TbCo}_{2}(5 \mathrm{~nm}) / \mathrm{FeCo}(5 \mathrm{~nm})$, was subsequently deposited by $\mathrm{RF}$ sputtering. The magneto-elastic constant $b^{\gamma, 2}$ is equal to $-7 \mathrm{MPa}$. A magnetic EA was obtained in the magnetic layer by applying a magnetic field during deposition. After removing the resist on the edges using acetone, this layer also served as top electrode.

Fig. 7 shows the considered device along with the magnetic field configuration used in the experiments. The same measurement setup was used for the characterizations.

We investigated the results given for vibrations at higher frequency modes. Fig. 8 shows the ME voltage amplitude measured as a function of the magnetic polarizing field $H_{S}$, when exciting the actuator at $35 \mathrm{KHz}$ with a $12.5 \mathrm{Oe}(1.25 \mathrm{mT})$ magnetic field amplitude. This mode has been identified as the first longitudinal mode of the actuator: no bending or torsion angle

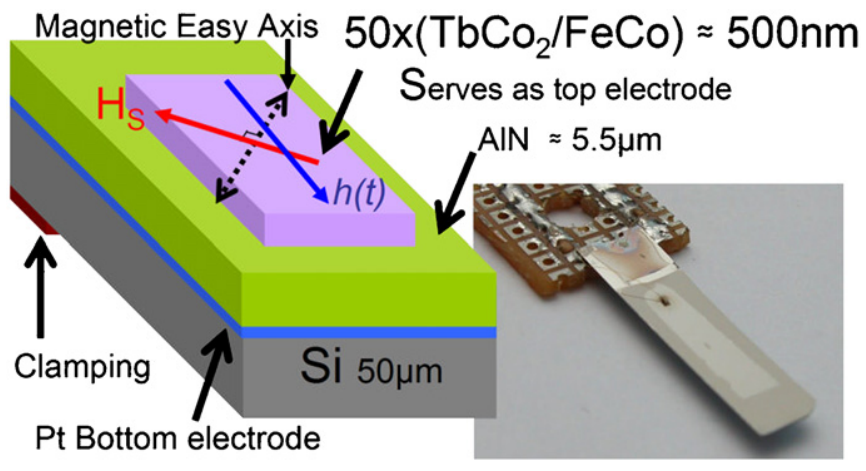

Fig. 7. Left: magnetic field configuration for the silicon cantilever coated with the AlN and magnetostrictive layers. $H_{s}$ is the polarizing field. $h(t)$ is the excitation field. Bottom: actual device.

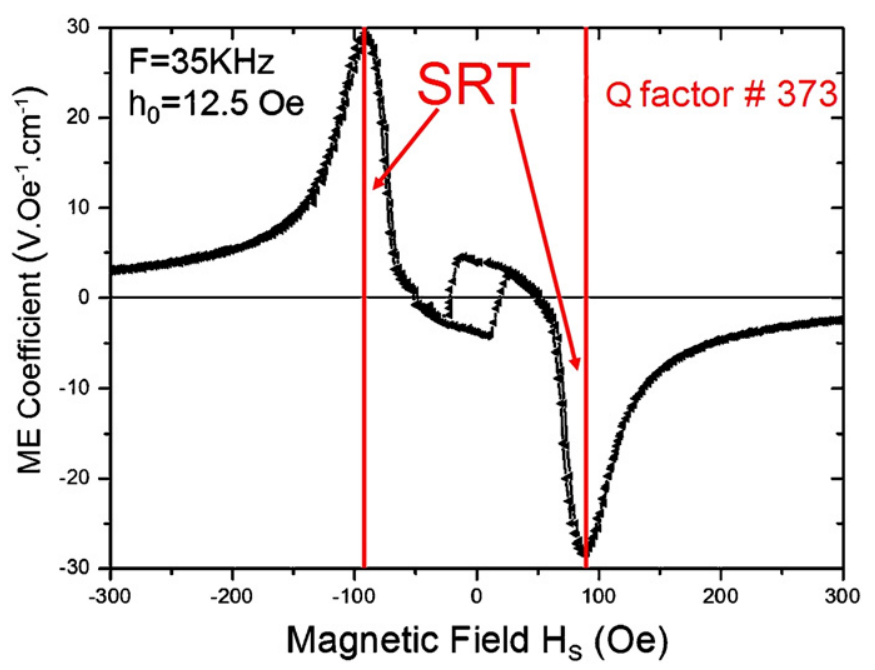

Fig. 8. ME voltage for the first longitudinal mode as a function of the polarizing field $H_{S}$.

could be measured optically. The effect of the SRT can also be clearly seen here. In resonant mode $30 \mathrm{~V} \mathrm{Oe}^{-1} \mathrm{~cm}^{-1}$ could be achieved with a measured $Q$ factor of 373 . Here, the $500 \mathrm{~nm}$ active magnetic layer is 11 times thinner than the AlN layer.

\section{Conclusions}

The ME effect in the considered structures is enhanced using the properties of the field induced SRT in the magnetostrictive nanostructured film. With bulk PZT substrates, ME coefficients of more than $580 \mathrm{mVOe}^{-1} \mathrm{~cm}^{-1}$ in resonance mode could be measured with magnetostrictive layer thicknesses of only $500 \mathrm{~nm}$. With magnetostrictive film on piezoelectric AlN film, a value of $30 \mathrm{VOe}^{-1} \mathrm{~cm}^{-1}$ was measured. These results bring possibilities and tools for the design of an integrated detection of vibrations in magnetostrictive actuators as well as high sensitivity magnetoelectric magnetic field sensors.

\section{Acknowledgements}

This work was supported by the European project Interreg IIIa number 198 and the Russian State contract no. 02.513.11.3357. 


\section{References}

[1] P. Curie, J. Phys. (Paris) III 3 (1894) 343.

[2] L.D. Landau, E.M. Lifshitz, Electrodynamics of Continuous Media, Pergamon Press, Oxford, 1960, p. 119 (Trans. of Russian ed., 1958).

[3] M. Fiebig, J. Phys. D Appl. Phys. 38 (2005) R123.

[4] N.A. Spaldin, M. Fiebig, Science 309 (2005) 391.

[5] S. Dong, J.F. Li, D. Viehland, Appl. Phys. Lett. 84 (2004) 4188

[6] J.G. Wan, Z.Y. Li, Y. Wang, M. Zeng, G.H. Wang, J. Liu, Appl. Phys. Lett. 86 (2005) 202504.

[7] H. Yu, M. Zeng, Y. Wang, J.G. Wan, J. Liu, Appl. Phys. Lett. 86 (2005) 032508.

[8] S.S. Guo, S.G. Lu, Z. Xu, X.Z. Zhao, S.W. Or, Appl. Phys. Lett. 88 (2006) 182906

[9] H. Zheng, J. Wang, S.E. Lofland, Z. Ma, L. Mohaddes-Ardabili, T. Zhao, L. Salamanca-Riba, S.R. Shinde, S.B. Ogale, F. Bai, D. Viehland, Y. Jia, D.G. Schlom, M. Wuttig, A. Roytburd, R. Ramesh, Science 303 (2004) 661.

[10] N. Tiercelin, J. Ben Youssef, V. Preobrazhensky, P. Pernod, H. Le Gall, J. Magn Magn. Mater. 249 (3) (2002) 519.

[11] H. Le Gall, J. Ben Youssef, F. Socha, N. Tiercelin, V. Preobrazhensky, P. Pernod, J. Appl. Phys. 87 (8) (2000).

[12] E. Quandt, A. Ludwig, D.G. Lord, C.A. Faunce, J. Appl. Phys. 83 (11) (1998).

[13] V. Ozhogin, V. Preobrazhensky, J. Magn. Magn. Mater. 100 (1991) 544.
[14] P. Pernod, V. Preobrazhensky, J. Magn. Magn. Mater. 185 (1) (1998) 127.

[15] V. Preobrazhensky, P. Pernod, J. Appl. Phys. 81 (8 PART 2B) (1997) 5709.

[16] N. Tiercelin, V. Preobrazhensky, P. Pernod, H. Le Gall, J. Ben Youssef, J. Magn. Magn. Mater. 210 (2000) 302.

[17] N. Tiercelin, P. Pernod, V. Preobrazhensky, H. Le Gall, Ben Youssef, Ultrasonics 38 (1) (2000) 64.

[18] N. Tiercelin, P. Pernod, Sensors Actuators A Physical 81 (1) (2000) 162.

[19] A. Debray, A. Ludwig, T. Bourouina, A. Asaoka, N. Tiercelin, G. Reyne, T. Oki, E. Quandt, H. Muro, H. Fujita, J. Microelectromechanical Syst. 13 (2) (2004) 264.

[20] L. Landau, E. Lifchitz, Course of Theoretical Physics: Statistical Physics, French ed., Ellipses, 1994.

[21] N. Tiercelin, V. Preobrazhensky, P. Pernod, A. Ostaschenko, APL 92 (2008) 062904.

[22] E. du Trémolet de Lacheisserie, J.C. Peuzin, J. Magn. Magn. Mater. 136 (1994) 189.

[23] J. Ben Youssef, N. Tiercelin, F. Petit, H. Le Gall, V. Preobrazhensky, P. Pernod, IEEE Trans. Magn. 38 (5) (2002) 2817.

[24] A. Ostaschenko, V. Preobrazhensky, P. Pernod, Phys. Solid State 50 (3) (2008) 446.

[25] V. Mortet, O. Elmazria, M. Nesladek, M.B. Assouar, G. Vanhoyland, J. D’Haen, M. D’Olieslaeger, P. Alnot, Appl. Phys. Lett. 81 (2002) 1720. 